\title{
Hover, Transition, and Level Flight Control Design for a Single-Propeller Indoor Airplane
}

\author{
Adrian Frank*, James S. McGrew† Mario Valenti; Daniel Levine§, and Jonathan P. How \\ Aerospace Controls Laboratory, Massachusetts Institute of Technology, Cambridge, MA, 02139
}

\begin{abstract}
This paper presents vehicle models and test flight results for an autonomous fixed-wing aircraft with the capability to take off, hover, transition to and from level-flight, and perch on a vertical landing platform. These maneuvers are all demonstrated in the highly space constrained environment of the Real-time indoor Autonomous Vehicle test ENvironment (RAVEN) at MIT. RAVEN promotes the rapid prototyping of UAV planning and control technologies by allowing the use of unmodified commercially available model aircraft for autonomous flight. Experimental results of several hover tests, transition maneuvers, and perch landings are presented. By enabling a fixed-wing UAV to achieve these feats, we demonstrate that the desirable speed and range performance of an autonomous fixed-wing aircraft in level flight can be complimented by hover capabilities that are typically limited to rotary-wing vehicles. This combination has the potential to significantly ease support and maintenance of operational autonomous vehicles.
\end{abstract}

\section{Introduction}

Unmanned aerial vehicles (UAVs) are being used to perform a wide variety of missions in an ever expanding range of operational environments. As UAV capabilities increase, so do the possibilities for practical application in the real world. The ability to hover would give a UAV certain flexibility with regards to operating environment and mission capabilities generally not possible with a traditional fixed-wing aircraft design. By enabling a fixed-wing UAV to perform transitions between hover and flight (and vice-versa) in a confined environment the desirable capabilities of rotary-wing and fixed-wing aircraft configurations can be realized in a single design. The UAV gains the ability to perform a multitude of missions previously limited to rotary-wing UAVs while maintaining the high speed cruise and extended range associated with fixed-wing UAVs. In an open environment, a fixed-wing UAV could fly a loiter pattern to remain on station for extended time periods, however, with the added spatial constraints imposed by most urban environments, a loiter pattern may not be feasible. Furthermore, missions such as reconnaissance, search and rescue, surveillance, and various other scenarios can sometimes benefit from having a sensory unit that can maintain a stationary position. Using fixed-wing UAVs for sensing missions in enclosed spaces can provide additional operational advantages, for example, a fixed-wing UAV can perform quick dashes from one position to another, allowing for quick relocation. The capability to hover

\footnotetext{
${ }^{*}$ Visiting Student, Department of Aeronautics and Astronautics, a_frank@mit.edu, M.Sc. Candidate, Department of Aeronautical and Vehicle Engineering, Royal Institute of Technology, Stockholm, Sweden

${ }^{\dagger}$ S.M. Candidate, Department of Aeronautics and Astronautics, jsmcgrew@mit.edu

${ }^{\ddagger}$ Ph.D., Department of Electrical Engineering and Computer Science, valenti@mit.edu, Student Member AIAA.

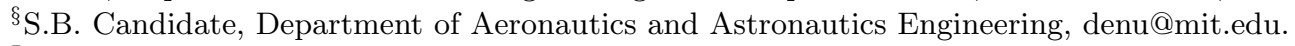

`Professor of Aeronautics and Astronautics, jhow@mit.edu, Associate Fellow AIAA.
} 
enables vertical take-offs and landings, which do not require runways or facilities with large spaces. These combined factors make fixed-wing UAVs with transition capabilities ideal for missions where spatial constraints are dominant.

The usefulness of transition capabilities in fixed-wing UAVs has been acknowledged by numerous research teams. The first successful manually controlled transitions were performed in 1954 with the Convair XFY-1 "Pogo". ${ }^{14}$ Currently, there are several research groups working to develop vehicles that possess the capability to fly in both a level-flight and hover configuration. A custom designed, radio-controlled $(\mathrm{R} / \mathrm{C})$ airplane was developed at Drexel University. The airplane is manuallycontrolled in level-flight operations and transitions to a computer-controlled hover configuration upon user input. The airplane has onboard processing and sensing using a commercially available internal measurement unit (IMU). ${ }^{13}$ At Brigham Young University (BYU) a trajectory generator has been used to simulate the actions of a commercially available R/C model of the Convair XFY1 to study the autonomous hover, flight, and transitions problem. ${ }^{8}$ Researchers at University of Sydney in Australia have designed and built a T-wing tail-sitter currently capable of autonomous hover. ${ }^{9}$ An on-line video presents the vehicle's capability to autonomously take-off from a vertical orientation, hover, transition to conventional flight, fly a path according to predefined waypoints, transition back to hover, and land. The vehicle uses onboard processing and sensing including GPS measurements for position sensing. ${ }^{11}$ Successful autonomous transitions from flight to hover (and vice-versa) have also been performed by researchers at Georgia Tech. Their experiments used a large, commercially available, $\mathrm{R} / \mathrm{C}$ airplane equipped with appropriate electronics. ${ }^{3}$

Although transitions from a traditional aircraft level-flight mode to the hover position (and vise-versa) have been discussed as a means of enabling missions to be performed in enclosed areas, the projects discussed above have all been undertaken in areas where space has not been a limiting factor. In previous research, autonomous take-offs and landings are generally performed either from a flat, open surface, or by hand-launch. This effectively limits the possibility to launch and land in an environment where space is limited or access is difficult. In addition, to the best of our knowledge the first reported instance of a fixed-wing autonomous UAV perching on vertical landing platform is shown in [12]. However, flight test data for this landing experiment was not provided in this video submission.

This paper presents both vehicle models and test flight results for an autonomous fixed-wing airplane that is designed to take-off to a hover, transition to and from traditional fixed-wing level flight modes, and perch on a vertical landing platform in a space-constrained environment. These test flights were performed indoors, thus introducing critical spatial constraints comparable to elements of an urban environment. A model of the fixed-wing airplane in both hover and level flight is presented, followed by descriptions of the control systems and guidance logic. In addition, this paper presents flight and perching results for a fixed-wing aircraft in hover configuration. Flight test results for the vehicle flying in the transition configuration and level flight mode are also provided. All of the flights provided in this paper were performed using the MIT Real-time indoor Autonomous Vehicle test ENvironment (RAVEN) and are available online at http://aerobatics.mit.edu

\section{Hardware and Control Structure}

The first objective of the project was to find a vehicle platform that could be used to study and implement technology that allows a fixed-wing airplane to hover, land/perch, transition and fly in an indoor environment and other heavily constrained spaces. Two different commercially-available off-the-shelf (COTS) R/C fixed-wing aircraft were used in these tests. First, the Blade 3D foam airplane was used for early flight testing due to its lightweight, low-cost and, durable airframe. Later, the Ikarus Yak 54 Shock Flyer foam airplane was selected for the final flight testing due to its better aerodynamic behavior and higher structural rigidity. Both airplanes have the capability 
to make the tight turns necessary to enable level flight and transitions in RAVEN's constrained flight space.

Using the architecture described in [7], each air vehicle can be controlled by a ground-based computer via the trainer port on an $\mathrm{R} / \mathrm{C}$ transmitter. In addition, the attitude and position of the vehicle can be sensed by a motion capture system by placing lightweight reflective markers on the vehicle. The markers reflect visible red light emitted from LED-arrays mounted on the cameras (see Figure 1). Images from several cameras are compared and the position and attitude of the vehicle is calculated based on a known geometry of markers on the vehicle. Therefore, all computations are done offboard, allowing the use of the vehicle in its off-the-shelf configuration, which enables rapid prototyping and cost effective testing. If desired, this architecture could be augmented by an onboard internal measurement unit (IMU) and processor for further indoor or outdoor autonomous operations.

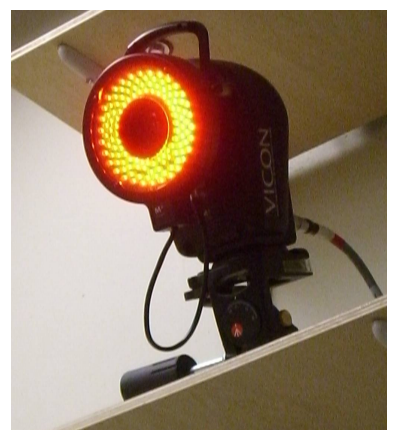

Figure 1. VICON motion capture camera. Lens located at center of the LED-array.

As shown in Figure 2, the control structure used for the airplane is that of an automaton with three states: land/perch, hover, and flight. Similar structures have been used in the past to control rotary-wing vehicles performing complex maneuvers with success. ${ }^{4}$ Hover is defined as a state in which the airplane maintains a steady or semi-steady position with the aircraft's nose perpendicular to the ground. The airplane can be given movement commands in hover. Level flight is a state in which the airplane's wings generate the main portion of the lift and the nose of the airplane is parallel to the ground.

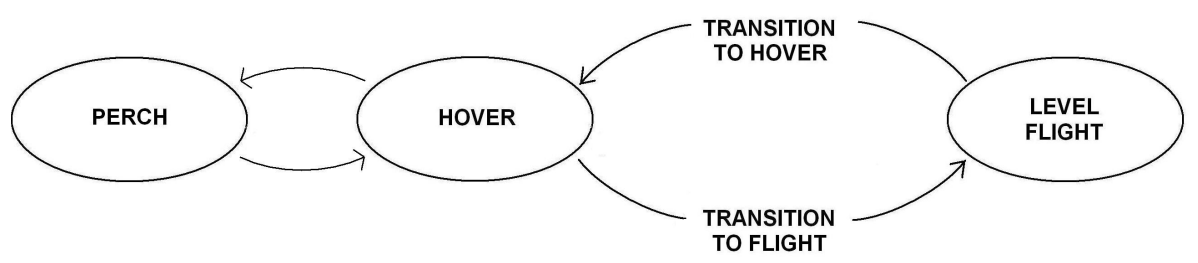

Figure 2. Automaton structure of the control system logic.

In each of our experiments, the airplane was able to take off horizontally from the ground or vertically from a perching stand before entering the hover state. While in hover, the airplane can be moved to any point in space and rolled to a specified orientation about the aircraft longitudinal axis. In addition, the vehicle can transition to and from the level flight mode, allowing the vehicle to move quickly between desired locations. The level flight state is maintained for as long as waypoints are available. As the airplane approaches the last defined level-flight waypoint, a transition to hover is initiated, causing the airplane to stay over the last waypoint until commanded otherwise. These three main parts of the control system are discussed in detail in this paper.

\section{Quaternion Attitude Description}

Modeling: In this paper, it is assumed that the Earth reference frame, $\left(x_{E}, y_{E}, z_{E}\right)$, is inertial. In addition, there exists a set of conversions between the Earth frame and the body frame, $\left(x_{B}\right.$, $\left.y_{B}, z_{B}\right)$, affixed to the airplane, assumed to be rigid. An illustration of the reference system used can be found in Figure 3 .

One of the true challenges of performing flights involving transitions is to have a global attitude reference that allows the vehicle's attitude to be described in a non-singular manner regardless of 
the attitude of the vehicle. There are several methods of describing the attitude of an airplane, the most common being the Euler angle representation. A primary benefit of using Euler angles is that the angles intuitively correspond to roll, pitch, and yaw. However, a deficiency inherent in the Euler angle description becomes clear as a vehicle approaches a pitch angle of $\pm 90^{\circ}$, when the Euler angle description fails to distinguish between roll and yaw. ${ }^{1}$ This singularity does not pose a problem as long as the vehicle's flight envelope is limited in pitch; as is the case in typical rotary-wing aerial vehicles where large pitch angles are undesirable during nominal operation. The assumption of a limited range of pitch angles also applies to most airplanes, where relatively straight and level flight is the only condition of interest. For this project, both hover and flight modes of an airplane are of interest. Therefore, a pitch angle-limited flight envelope is unfeasible, and a singularity-free description of an arbitrary attitude is necessary.

To avoid the singularity exhibited by an Euler angle attitude description, quaternions are used as a global attitude reference. ${ }^{6}$ A quaternion is a 4 tuple of real numbers of unit length, which limits it to three degrees of freedom. ${ }^{6}$ A quaternion can also be written as a rotation by an angle $\zeta / 2$ about a three-dimensional unit-vector $\bar{E}$, such that

$$
q=\left[\begin{array}{c}
\cos (\zeta / 2) \\
\bar{E} \sin (\zeta / 2)
\end{array}\right]=\left[\begin{array}{c}
q_{0} \\
q_{1} \\
q_{2} \\
q_{3}
\end{array}\right]
$$
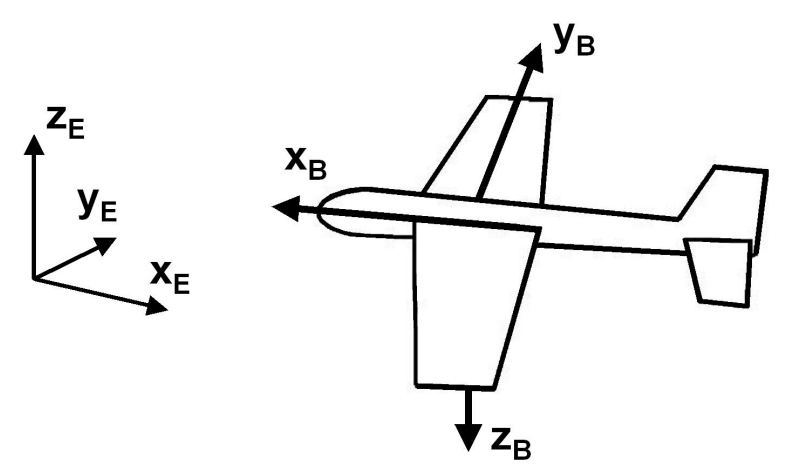

Figure 3. Reference frame and body frame

In addition, we define the zero-quaternion $q^{(0)}=[1,0,0,0]^{T}$ to be a reference attitude corresponding to an Euler angle description of $\phi=\theta=\psi=0^{\circ}$. Where $\phi, \theta$, and $\psi$ are rotations about $x_{B}, y_{B}$, and $z_{B}$ applied in reverse order, that is, starting by rotating about $z_{B}$ with the angle $\psi$. The zero-quaternion here refers to the attitude in which the nose of the airplane is pointed straight North, with the airplane in level flight.

Two key concepts are used in the following: quaternion multiplication and quaternion splitting. A quaternion can be thought of as a rotation from the zero-quaternion to the current attitude, which can be represented as a matrix rotation about a vector. Quaternion multiplications are successive rotations applied to a zero-quaternion that are non-commutative, and possess laws similar to those of matrix multiplications. Quaternion multiplication is defined $\operatorname{as}^{5,6}$

$$
p \star q=M(p) \cdot q=M(p) \cdot M(q) \cdot\left[\begin{array}{l}
1 \\
0 \\
0 \\
0
\end{array}\right]
$$

where $p$ and $q$ are quaternions and $M(p)$ and $M(q)$ are quaternion matrices, defined as

$$
M(\eta)=\left[\begin{array}{cccc}
\eta_{0} & -\eta_{1} & -\eta_{2} & -\eta_{3} \\
\eta_{1} & \eta_{0} & -\eta_{3} & \eta_{2} \\
\eta_{2} & \eta_{3} & \eta_{0} & -\eta_{1} \\
\eta_{3} & -\eta_{2} & \eta_{1} & \eta_{0}
\end{array}\right]
$$

The inverse of the quaternion matrix also follows the rules of matrix multiplication. Since quaternions have a unit length, no scaling occurs and $M^{-1}=M^{T}$. 
In controlling a vehicle, the current attitude $q^{\text {current }}$ is not necessarily of interest, but rather the deviation, or error, $q^{\text {dev }}$ from a desired reference attitude $q^{\text {ref }}$. A deviation quaternion can be defined using quaternion multiplication as

$$
q^{\text {current }}=q^{\mathrm{dev}} \star q^{\mathrm{ref}}
$$

Given this definition of a deviation from the reference state, a quaternion split is specified as

$$
q^{\mathrm{dev}}=q^{x} \star q^{y} \star q^{z} .
$$

The three quaternions resulting from (3) are defined as separate rotations about the $x_{B^{-}}, y_{B^{-}}$and $z_{B}$-axis, respectively, applied in reverse order. Consequently, these quaternions represent the rotations performed in an Euler angle description, where components of quaternions $q^{x}=\left[q_{0}^{x}, q_{1}^{x}, 0,0\right]^{T}$, $q^{y}=\left[q_{0}^{y}, 0, q_{2}^{y}, 0\right]^{T}$, and $q^{z}=\left[q_{0}^{z}, 0,0, q_{3}^{z}\right]^{T}$ are defined as

$$
\begin{array}{ll}
q_{0}^{x}=\cos (\phi)=\left(1-2\left(q_{1}^{2}+q_{2}^{2}\right)\right) / q_{0}^{y}, & q_{1}^{x}=\sin (\phi)=\left(2\left(q_{0} q_{1}+q_{2} q_{3}\right)\right) / q_{0}^{y}, \\
q_{0}^{y}=\cos (\theta)=\sqrt{1-4\left(q_{0} q_{2}-q_{1} q_{3}\right)^{2}}, & q_{2}^{y}=\sin (\theta)=2\left(q_{0} q_{2}-q_{1} q_{3}\right), \\
q_{0}^{z}=\cos (\psi)=\left(1-2\left(q_{2}^{2}+q_{3}^{2}\right)\right) / q_{0}^{y}, & q_{3}^{z}=\sin (\psi)=\left(2\left(q_{1} q_{2}+q_{0} q_{3}\right)\right) / q_{0}^{y} .
\end{array}
$$

Although split quaternions present an intuitive way of working with quaternions, it should be noted that the split defined by (3) is not singularity free. In particular, since the split quaternions in (3) correspond to Euler angles, the singularity at a pitch of $\pm 90^{\circ}$ is re-introduced. However, in the control laws used in this paper, the quaternion split is only used for control error, or more specifically, the quaternion deviation as defined by (2).

Throughout the flight envelope, a global quaternion based reference frame is used to keep track of the attitude of the aircraft. The attitude of the aircraft using this global reference is denoted $q^{\text {current }}$ in (2), which can be regarded as the deviation from the zero-quaternion. During hover, the quaternion deviation is computed using the current desired reference attitude (essentially vertical). During the transition from hover to level flight, the desired reference attitude is still given as a vertical attitude, but a position offset is commanded to the waypoint follower to force the vehicle to pitch the nose down (see Section VI for a detailed discussion). To ensure that the magnitude of the pitch error will always be less than $90^{\circ}$ during a transition from hover to level flight, the controller mode changes into the level flight state as the pitch angle drops below $30^{\circ}$ (pitch angle of $0^{\circ}$ corresponds to an attitude where the longitudinal axis of the airplane is in the horizontal plane). The desired reference of the level flight controller is then based on the requirements to maintain horizontal flight (again see Section VI for details). Note that in the event that the pitch error should exceed $60^{\circ}$, the controller will change from the level flight state to hover. Due to the potential hazard of an arbitrary transition to level flight, the airplane always remains in the hover state until a transition is enabled by the operator.

\section{Airplane in Hover}

Modeling: Since the airplane used in this research has only one propeller, a throttle input will produce both thrust and a moment due to rotation of the propeller. The propeller moment comes from two main contributors. The first contributor is the moment created by a change in propeller speed. This propeller acceleration moment is calculated as

$$
M_{\mathrm{acc}}=I_{\text {prop }} \dot{\omega}
$$


where $I_{\text {prop }}$ is the moment of inertia of the propeller defined in the $x_{B}$-direction. The moment $M_{\text {acc }}$ about the airframe $x_{B}$-axis is created by the angular acceleration of the propeller, $\dot{\omega}$.

Propeller drag is the second contributor to the total propeller moment and can be estimated using the NACA-standardized thrust and power coefficients $C_{T}$ and $C_{P}{ }^{2}$

$$
\begin{aligned}
C_{T} & =\frac{T}{\rho n^{2} d^{4}} \\
C_{P} & =\frac{P}{\rho n^{3} d^{5}}
\end{aligned}
$$

In equations (5) and (6) the thrust $T=m g$ is determined using Newton's second law, where the thrust is the force necessary to keep the airplane in a sustained hover, that is, the mass of the airplane $m$ times the gravitational acceleration $g$. In this derivation, it is assumed the thrust generated by the propeller is the only force counteracting gravity. The flow over the propeller is assumed to be essentially incompressible, and hence, the density of the air $\rho$ is constant. The remaining parameters are the diameter of the propeller $d$, and the rotational speed of the propeller in revolutions per second, $n$. With a known thrust coefficient, the rotational speed of the propeller can be estimated using (5). The moment created by propeller drag can then be estimated using the relationship for power, $P=M_{\mathrm{drag}} 2 \pi n$. The moment can be expressed as

$$
M_{\mathrm{drag}}=\frac{T d C_{P}}{2 \pi C_{T}} .
$$

During hover, the control surfaces are only partially covered by the airflow from the propeller. Utilizing actuator disk theory, the velocity of the propeller airflow at the control surfaces can be estimated. With the additional assumptions of steady flow, discontinuous pressure, and continuous velocity, the velocity of the propeller airflow at the control surfaces in hover can be estimated using Bernoulli's equation as

$$
u_{\text {prop }}=\sqrt{\frac{2 T}{\rho A_{\text {disk }}}},
$$

where $u_{\text {prop }}$ is the axial velocity of the propeller airflow, and $A_{\text {disk }}=\pi d^{2} / 4$ is the area of the actuator disk representing the spinning propeller.

The lift coefficient derivatives for the ailerons, elevator, and rudder, $C_{L_{\delta_{\text {ail }}}}, C_{L_{\delta_{\text {ele }}}}$, and $C_{L_{\delta_{\text {rud }}}}$, are determined using flat plate approximations of the control surfaces. These approximations are predicated on airplane geometry. Lift produced by the wings can be calculated as

$$
L_{\text {wing }}=\frac{1}{2} \rho u_{\infty}^{2} A_{\text {wing }} C_{L_{\alpha}} \alpha .
$$

Again, a flat plate approximation of the wing lift coefficient derivative $C_{L_{\alpha}}$ is valid because of airfoil geometry. The angle of attack can, using small angle approximation, be described as $\alpha=\dot{z}_{B} / u_{\infty}$.

While in a hover, aileron deflection does not greatly affect roll. Although the deflection of the ailerons necessary to maintain a desired heading is considerable, a significant amount of drag is produced. This drag contribution can be modeled as

$$
D_{\text {ail }}=\left(C_{D, 0}+\frac{C_{L_{\delta_{\text {ail }}}}^{2} \delta_{\text {ail }}^{2}}{\pi e A R}\right) \frac{1}{2} \rho u_{\text {prop }}^{2} A_{\text {aileron,prop }}
$$

As a pitching or yawing motion is induced to the airframe, the propeller airflow creates an apparent angle of attack of the control surfaces. This apparent angle of attack in turn causes 
damping moments in the aircraft pitch and yaw dynamics. The damping due to propeller airflow lag can be modeled as

$$
\begin{aligned}
q_{\text {damp }} & =L_{\text {elevator }} \alpha_{\text {elevator }} A_{\text {elevator }} \frac{1}{2} \rho u_{\text {prop }}^{2} \\
r_{\text {damp }} & =L_{\text {rudder }} \alpha_{\text {rudder }} A_{\text {rudder }} \frac{1}{2} \rho u_{\text {prop }}^{2} \\
\alpha_{\text {elevator }} & =\frac{L_{\text {prop2elevator }} q}{u_{\text {prop }}} \\
\alpha_{\text {rudder }} & =\frac{L_{\text {prop2rudder }} r}{u_{\text {prop }}} \\
C_{\text {ele }_{\mathrm{d}}} & =\frac{q_{\text {damp }}}{q u_{\text {prop }}} \\
C_{\text {rud }_{\mathrm{d}}} & =\frac{r_{\text {damp }}}{r u_{\text {prop }}}
\end{aligned}
$$

where $q_{\text {damp }}$ and $r_{\text {damp }}$ are the pitch and roll damping moments, $L_{\text {prop2elevator }}$ and $L_{\text {prop2rudder }}$ are the distances from the propeller to the elevator and rudder, and $\alpha_{\text {elevator }}$ and $\alpha_{\text {rudder }}$ are the apparent angles of attack, respectively. Separating variables from constants, the dimensional elevator and rudder drag constants $C_{\text {ele }_{\mathrm{d}}}$ and $C_{\text {rud }_{\mathrm{d}}}$ can be defined as above.

Based on the model developed so far, the general equations of motion for an airplane can be expressed using a quaternion attitude description as

$$
\begin{gathered}
x_{E}=2\left(q_{1} q_{2}+q_{0} q_{3}\right) x_{B}+\left[1-2\left(q_{1}^{2}+q_{3}^{2}\right)\right] y_{B}+2\left(q_{2} q_{3}-q_{0} q_{1}\right) z_{B} \\
y_{E}=\left[1-2\left(q_{2}^{2}+q_{3}^{2}\right)\right] x_{B}+2\left(q_{1} q_{2}-q_{0} q_{3}\right) y_{B}+2\left(q_{0} q_{2}+q_{1} q_{3}\right) z_{B} \\
z_{E}=-2\left(q_{1} q_{3}-q_{0} q_{2}\right) x_{B}-2\left(q_{0} q_{1}+q_{2} q_{3}\right) y_{B}-\left[1-2\left(q_{1}^{2}+q_{2}^{2}\right)\right] z_{B} \\
\ddot{x}_{B}=2\left(q_{1} q_{3}-q_{0} q_{2}\right) g+\frac{1}{m} \delta_{\text {throttle }}-\left(C_{D, 0}+\frac{C_{L}^{2}}{\pi e A}\right) \frac{1}{2} \rho u_{\infty}^{2} A_{\text {wing }}-\frac{D_{\text {ail }}}{m} \\
\ddot{y}_{B}=2\left(q_{0} q_{1}+q_{2} q_{3}\right) g+C_{L_{\delta_{\text {rud }}}} \frac{1}{2} \rho\left(u_{\text {prop }}^{2} A_{\text {rudder,prop }}+u_{\infty}^{2} A_{\text {rudder }}\right) \delta_{\text {rudder }} \\
\ddot{z}_{B}=\left[1-2\left(q_{1}^{2}+q_{2}^{2}\right)\right] g+C_{L_{\delta_{\text {ele }}}} \frac{1}{2} \rho\left(u_{\text {prop }}^{2} A_{\text {elevator,prop }}+u_{\infty}^{2} A_{\text {elevator }}\right) \delta_{\text {elevator }}-L_{\text {wing }} \\
\dot{p}=\quad q r\left(\frac{I_{y}-I_{z}}{I_{x}}\right)+C_{L_{\delta_{\text {ail }}}} \frac{1}{2} \rho\left(u_{\text {prop }}^{2} A_{\text {aileron,prop }} L_{\text {aileron,prop }}+u_{\infty}^{2} A_{\text {aileron }} L_{\text {aileron }}\right) \frac{1}{I_{x}} \delta_{\text {aileron }} \\
\dot{q}=\operatorname{pr}\left(\frac{I_{z}-I_{x}}{I_{y}}\right)+C_{L_{\delta_{\text {ele }}}} \frac{1}{2} \rho\left(u_{\text {prop }}^{2} A_{\text {elevator,prop }}+u_{\infty}^{2} A_{\text {elevator }}\right) \frac{L_{\text {elevator }}}{I_{y}} \delta_{\text {elevator }}-\frac{C_{\text {ele }} u_{\text {prop }} q}{I_{y}} \\
\dot{r}=\operatorname{pr}\left(\frac{I_{x}-I_{y}}{I_{z}}\right)+C_{L_{\delta_{\text {rud }}}} \frac{1}{2} \rho\left(u_{\text {prop }}^{2} A_{\text {rudder,prop }}+u_{\infty}^{2} A_{\text {rudder }}\right) \frac{L_{\text {rudder }}}{I_{z}} \delta_{\text {rudder }}-\frac{C_{\text {rud }_{d}} u_{\text {prop }} r}{I_{z}}
\end{gathered}
$$




$$
\begin{aligned}
\dot{q}_{0} & =\frac{1}{2}\left(-p q_{1}-q q_{2}-r q_{3}\right) \\
\dot{q}_{1} & =\frac{1}{2}\left(p q_{0}-q q_{3}+r q_{2}\right) \\
\dot{q}_{2} & =\frac{1}{2}\left(p q_{3}+q q_{0}-r q_{1}\right) \\
\dot{q}_{3} & =\frac{1}{2}\left(-p q_{2}+q q_{1}+r q_{0}\right)
\end{aligned}
$$

where the vector $\left[q_{0}, q_{1}, q_{2}, q_{3}\right]^{T}$ is the attitude quaternion. The variables $A_{\text {aileron,prop }}, A_{\text {elevator,prop }}$, $A_{\text {rudder,prop }}, A_{\text {aileron }}, A_{\text {elevator }}$, and $A_{\text {rudder }}$ are the areas of the control surfaces covered by the propeller airflow and the total areas of the control surfaces affected by freestream airflow, respectively. The freestream airflow velocity is conventionally denoted as $u_{\infty}$. The lengths $L_{\text {aileron,prop }} L_{\text {aileron }}$, $L_{\text {elevator }}$, and $L_{\text {rudder }}$ are the moment arms of the various control surfaces.

Drag is calculated using the induced drag coefficient $C_{D i}=\frac{C_{L}^{2}}{\pi e R}$ and parasitic drag coefficient $C_{D, 0}$, where $C_{L}=C_{L_{\alpha}} \alpha$ is the lift coefficient of the wing, $e$ is the wing efficiency, and $R$ is the aspect ratio of the wing. The drag acting on the airplane while in a hover condition is negligible. This assumption can be made since the freestream velocity is very low. The force terms in the $y_{B^{-}}$ and $z_{B}$-axis due to control surface deflection are small in hover, again due to very low freestream velocity, and can hence be disregarded.

Due to symmetry of the airframe, the cross-coupled inertia terms $I_{x y}=I_{y z}=0$. Additionally, the term $I_{x z}$ is considerably smaller than diagonal terms of the inertia tensor $\left(I_{x}, I_{y}\right.$, and $\left.I_{z}\right)$ and is hence disregarded. Since the moment of inertia of the propeller $I_{\text {prop }}$ is considerably smaller than $I_{x}$, the propeller torque due to change in rotational speed of the propeller is negligible even when rapid changes in motor speed occur. All rotational speeds $p, q$, and $r$ are expected to be small enough such that products thereof can be disregarded without impacting the model accuracy.

The speed of the axial propeller airflow is considered constant while the airplane is in a hover state. It is also known that $u_{\infty}=0$ when the vehicle is stationary, and therefore

$$
\begin{aligned}
\delta_{\text {aileron }} & =\frac{2}{C_{L_{\delta_{\text {ail }}}} \rho u_{\text {prop }}^{2} A_{\text {aileron,prop }} L_{\text {aileron,prop }}} M_{\text {drag }}+\hat{\delta}_{\text {aileron }} \\
\delta_{\text {throttle }} & =m g+D_{\text {ail }}+\hat{\delta}_{\text {throttle }}
\end{aligned}
$$

where $\hat{\delta}_{\text {throttle }}$ is a small deviation from the expected throttle input necessary to maintain hover. The contribution of aileron drag to throttle setting will always be considerably less than that of gravity. Consequently, the aileron drag can be neglected in the horizontal movement terms.

A deviation quaternion can be calculated by projecting the current quaternion onto the product of a heading quaternion $q^{h d g}=[\cos (h d g / 2), \sin (h d g / 2), 0,0]^{T}=[\bar{c}, \bar{s}, 0,0]$, where $h d g$ is the heading reference, and a vertical quaternion $q^{v}=\left[\frac{\sqrt{2}}{2}, 0, \frac{\sqrt{2}}{2}, 0\right]^{T}$. The deviation quaternion represents the attitude error from the reference quaternion (in this case the vertical quaternion) to the attitude in which the airplane is currently hovering.

$$
\begin{aligned}
q^{\text {current }}=q^{\mathrm{dev}} \star q^{h d g} \star q^{v} \\
=\frac{\sqrt{2}}{2}\left[\begin{array}{r}
\bar{c} q_{0}^{\mathrm{dev}}-\bar{s} q_{1}^{\mathrm{dev}}-\bar{c} q_{2}^{\mathrm{dev}}+\bar{s} q_{3}^{\mathrm{dev}} \\
\bar{s} q_{0}^{\mathrm{dev}}+\bar{c} q_{1}^{\mathrm{dev}}+\bar{s} q_{2}^{\mathrm{dev}}+\bar{c} q_{3}^{\mathrm{dev}} \\
\bar{c} q_{0}^{\mathrm{dev}}-\bar{s} q_{1}^{\mathrm{dev}}+\bar{c} q_{2}^{\mathrm{dev}}-\bar{s} q_{3}^{\mathrm{dev}} \\
-\bar{s} q_{0}^{\mathrm{dev}}-\bar{c} q_{1}^{\mathrm{dev}}+\bar{s} q_{2}^{\mathrm{dev}}+\bar{c} q_{3}^{\mathrm{dev}}
\end{array}\right]
\end{aligned}
$$

The deviation from the desired state is expected to be small. Consequently, all products of deviated quaternion components other than $q_{0}^{\mathrm{dev}}$ can be disregarded (as $q_{0}^{\mathrm{dev}}$ is near unity when deviation is 
small). Using deviated quaternions, the simplified quaternion model can be written as

$$
\begin{aligned}
& \ddot{x}_{B}=\frac{1}{m} \hat{\delta}_{\text {throttle }} \\
& \ddot{y}_{B}=g q_{3}^{\mathrm{dev}} \\
& \ddot{z}_{B}=-g q_{2}^{\mathrm{dev}} \\
& \dot{p}=C_{L_{\delta_{\text {ail }}}} \frac{1}{2} \rho u_{\text {prop }}^{2} A_{\text {aileron,prop }} L_{\text {aileron,prop }} \frac{1}{I_{x}} \hat{\delta}_{\text {aileron }} \\
& \dot{q}=C_{L_{\delta_{\text {ele }}}} \frac{1}{2} \rho u_{\text {prop }}^{2} A_{\text {elevator,prop }} \frac{L_{\text {elevator }}}{I_{y}} \delta_{\text {elevator }}-\frac{C_{\text {ele }_{\mathrm{d}}} u_{\text {prop }} q}{I_{y}} \\
& \dot{r}=C_{L_{\delta_{\text {rud }}}} \frac{1}{2} \rho u_{\text {prop }}^{2} A_{\text {rudder,prop }} \frac{L_{\text {rudder }}}{I_{z}} \delta_{\text {rudder }}-\frac{C_{\text {rudd }_{\mathrm{d}}} u_{\text {prop }} r}{I_{z}} \\
& \dot{q}^{\operatorname{dev}}=\left[\begin{array}{cccc}
0 & \frac{p}{2} & \frac{q}{2} & \frac{r}{2}
\end{array}\right]^{T}
\end{aligned}
$$

The simplified model can be separated into two fourth-order and two second-order decoupled linear time-invariant (LTI) systems. The fourth-order systems describe the dynamic behavior of pitch and $z_{B}$-position, and yaw and $y_{B}$-position, respectively. The pitch- $z_{B}$ loop is affected by the elevator input, whereas the yaw- $y_{B}$ loop is affected by rudder input. The second order systems describe the $x_{B}$ loop and roll loop, respectively. Here, throttle controls altitude and the ailerons control roll. It can be noted that when the airplane is near hover, and has a heading reference of $0^{\circ}$, the system maps approximately to $x_{E}=y_{B}, y_{E}=z_{B}$, and $z_{E}=x_{B}$.

Hover Control: The hover controller uses full state feedback from the sensing system, which is made possible by accurate measurement data being available at $100 \mathrm{~Hz}{ }^{7}$ In order to improve position-keeping, integrators are incorporated into all four control schemes. The controller gains are optimized using linear quadratic (LQ) control techniques. Large penalties are assigned to pitch, roll, and their respective derivatives to ensure that the vehicle maintains a hover attitude. Small penalties on the position deviations prevent the vehicle from making sudden movements should the position change. The penalty on altitude deviation is large to ensure that the airplane accurately maintains an altitude, facilitating perching.

Due to the low structural rigidity of foam airplanes, quick control surface actuation will cause the airframe to twist. This twisting moment can be perceived by the motion capture system as a movement of the airplane, adding noise to the position and attitude measurements. This undesirable effect is partially mitigated by the fact that the servos cannot instantly deflect the control surfaces. Since the servo time constants are small enough to not completely resolve the issue, the LQ design is focused on minimizing rapid changes in control surface position. The use of the Ikarus Yak 54 Shock Flyer also helped to reduce the twisting effects.

\section{Level Flight}

Modeling: The level flight controller could use the global quaternion attitude reference for determining the aircraft orientation in a manner similar to that of the hover controller. However, it is simpler and more intuitive to develop the level flight model and controller using the standard Euler angle representation. The Euler angles are calculated from the global quaternion attitude reference to ensure consistency between the attitude descriptions used for hover and level flight. The conversion is straight forward and can be found in [6]. In order to control the vehicle, we first 
introduce the full equations of motion of the airplane in Euler angles.

$$
\begin{aligned}
& x_{E}=\cos \theta \sin \psi x_{B}+(\cos \phi \cos \psi+\sin \phi \sin \theta \sin \psi) y_{B} \\
& +(-\sin \phi \cos \psi+\cos \phi \sin \theta \sin \psi) z_{B} \\
& y_{E}=(\cos \theta \cos \psi) x_{B}+(-\cos \phi \sin \psi+\sin \phi \sin \theta \cos \psi) y_{B} \\
& +(\sin \phi \sin \psi+\cos \phi \sin \theta \cos \psi) z_{B} \\
& z_{E}=\sin \theta x_{B}-\sin \phi \cos \theta y_{B}-\cos \phi \cos \theta z_{B} \\
& \ddot{x}_{B}=-\sin \theta g+\frac{1}{m} \delta_{\text {throttle }}-\left(C_{D, 0}+\frac{C_{L}^{2}}{\pi e \AA R}\right) \frac{1}{2} \rho u_{\infty}^{2} A_{\text {wing }}-\frac{D_{\text {ail }}}{m} \\
& \ddot{y}_{B}=\sin \phi \cos \theta g+C_{L_{\delta_{\text {rud }}}} \frac{1}{2} \rho\left(u_{\text {prop }}^{2} A_{\text {rudder,prop }}+u_{\infty}^{2} A_{\text {rudder }}\right) \delta_{\text {rudder }} \\
& \ddot{z}_{B}=\cos \phi \cos \theta g+C_{L_{\delta_{\text {ele }}}} \frac{1}{2} \rho\left(u_{\text {prop }}^{2} A_{\text {elevator,prop }}+u_{\infty}^{2} A_{\text {elevator }}\right) \delta_{\text {elevator }}-L_{\text {wing }} \\
& \dot{p}=q r\left(\frac{I_{y}-I_{z}}{I_{x}}\right)+C_{L_{\delta_{\text {ail }}}} \frac{1}{2} \rho\left(u_{\text {prop }}^{2} A_{\text {aileron,prop }} L_{\text {aileron,prop }}+u_{\infty}^{2} A_{\text {aileron }} L_{\text {aileron }}\right) \frac{1}{I_{x}} \delta_{\text {aileron }} \\
& -\frac{M_{\mathrm{acc}}+M_{\mathrm{drag}}}{I_{x}} \\
& \dot{q}=\operatorname{pr}\left(\frac{I_{z}-I_{x}}{I_{y}}\right)+C_{L_{\delta_{\text {ele }}}} \frac{1}{2} \rho\left(u_{\text {prop }}^{2} A_{\text {elevator,prop }}+u_{\infty}^{2} A_{\text {elevator }}\right) \frac{L_{\text {elevator }}}{I_{y}} \delta_{\text {elevator }}-\frac{C_{\text {ele }_{\mathrm{d}}} u_{\text {prop }} q}{I_{y}} \\
& \dot{r}=p q\left(\frac{I_{x}-I_{y}}{I_{z}}\right)+C_{L_{\delta_{\text {rud }}}} \frac{1}{2} \rho\left(u_{\text {prop }}^{2} A_{\text {rudder }, \text { prop }}+u_{\infty}^{2} A_{\text {rudder }}\right) \frac{L_{\text {rudder }}}{I_{z}} \delta_{\text {rudder }}-\frac{C_{\text {rud }_{\mathrm{d}}} u_{\text {prop }} r}{I_{z}} \\
& \dot{\phi}=p+(q \sin \phi+r \cos \phi) \tan \theta \\
& \dot{\theta}=q \cos \phi-r \sin \phi \\
& \dot{\psi}=(q \sin \phi+r \cos \phi) \frac{1}{\cos \theta}
\end{aligned}
$$

Unlike an airplane in hover, the airplane in level flight mode follows a predefined trajectory. Hence, a description of position derivatives $\left(\ddot{x}_{B}\right.$ and $\left.\ddot{y}_{B}\right)$ does not necessarily clarify the airplane's dynamic behavior in the level flight condition. Therefore, a modification of the hover model of the airplane $(17-20)$ is required for level flight control. The trajectory follower used during level flight provides the controller with a lateral acceleration necessary to keep the vehicle on the desired trajectory. Due to the provided reference, a more intuitive way of considering the airplane dynamics is by looking at forward velocity $v_{x_{B}}$, altitude $z_{E}$, and lateral acceleration $a_{\text {lat }}$ rather than a position in the earth frame of reference.

For level flight, the pitch angle can be approximated using small angle approximations for sufficiently large velocities, i.e. not a stalled configuration. The pitch angle necessary to maintain an altitude in level flight, that is, to create sufficient lift to counteract the gravitational force on the airplane, is

$$
\theta_{\text {flight }}=\frac{2 m g}{\rho u_{\infty}^{2} C_{L_{\text {wing }}} A_{\text {wing }}+\delta_{\text {throttle }}} .
$$

Based on airplane geometry, the wing lift coefficient $C_{L_{\text {wing }}}$ can be estimated using flat plate approximation. It can furthermore be assumed that the aileron deflection will be small during level flight, due to the increased airflow over the ailerons and the increase in wetted aileron area. The reduction 
in aileron deflection compared to hover aileron deflection renders the aileron drag component $D_{\text {ail }}$ unnecessary.

Now we define

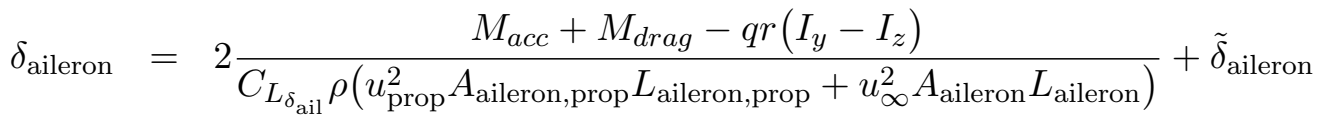

$$
\begin{aligned}
& \delta_{\text {elevator }}=2 \frac{-p r\left(I_{z}-I_{x}\right)}{C_{L_{\delta_{\text {ele }}}} \rho\left(u_{\text {prop }}^{2} A_{\text {elevator,prop }}+u_{\infty}^{2} A_{\text {elevator }}\right) L_{\text {elevator }}}+\tilde{\delta}_{\text {elevator }} \\
& \delta_{\text {rudder }}=2 \frac{-p q\left(I_{x}-I_{y}\right)}{C_{L_{\delta_{\text {rud }}} \rho\left(u_{\text {prop }}^{2} A_{\text {rudder,prop }}+u_{\infty}^{2} A_{\text {rudder }}\right) L_{\text {rudder }}}}+\tilde{\delta}_{\text {rudder }} \\
& \delta_{\text {throttle }}=m\left(C_{D, 0}+\frac{C_{L}^{2}}{\pi e R}\right) \frac{1}{2} \rho u_{\infty}^{2} A_{\text {wing }}+2 m g \sin \theta+\tilde{\delta}_{\text {throttle }}
\end{aligned}
$$

Altitude changes of the airplane are made mainly by varying the pitch angle of the airplane and thereby pointing the velocity vector slightly upward or downward. Due to this behavior, the altitude is a function of forward velocity and pitch angle as

$$
\dot{z}_{E}=v_{x_{B}} \sin \left(\theta-\theta_{\text {flight }}\right) .
$$

The forward velocity during level flight can be considered a semi-steady state, where different velocities are possible, but with few changes of reference velocity. Hence, we can define $v_{x_{B}}=u_{\infty}$. Note that this relationship is used only for altitude control. The assumption of a semi-steady velocity also promotes the description of $u_{\text {prop }}$ as a set speed, as well as a small angle approximation for $\theta$.

In the same manner as the forward velocity, the roll angle can be considered a semi-steady state. Hence, a desired roll angle $\phi_{\text {ref }}$ and desired pitch angle $\theta_{\text {ref }}$ can be defined and used to determine the equations of motion for set roll and pitch angles.

The simplified equations of motion for the airplane in level flight can be written as

$$
\begin{aligned}
& \dot{v}_{x_{B}}=\frac{1}{m} \tilde{\delta}_{\text {throttle }} \\
& a_{\text {lat }}=\left(q \sin \phi_{\text {ref }}+r \cos \phi_{\text {ref }}\right) u_{\infty} \\
& \dot{z}_{E}=\left(\theta-\theta_{\text {flight }}\right) u_{\infty} \\
& \dot{p}=C_{L_{\delta_{\text {ail }}}} \frac{1}{2} \rho\left(u_{\text {prop }}^{2} A_{\text {aileron,prop }} L_{\text {aileron,prop }}+u_{\infty}^{2} A_{\text {aileron }} L_{\text {aileron }}\right) \frac{1}{I_{x}} \tilde{\delta}_{\text {aileron }} \\
& \dot{q}=C_{L_{\delta_{\text {ele }}}} \frac{1}{2} \rho\left(u_{\text {prop }}^{2} A_{\text {elevator,prop }}+u_{\infty}^{2} A_{\text {elevator }}\right) \frac{L_{\text {elevator }}}{I_{y}} \tilde{\delta}_{\text {elevator }}-\frac{C_{\text {ele }_{\mathrm{d}}} u_{\text {prop }} q}{I_{y}} \\
& \dot{r}=C_{L_{\delta_{\text {rud }}}} \frac{1}{2} \rho\left(u_{\text {prop }}^{2} A_{\text {rudder,prop }}+u_{\infty}^{2} A_{\text {rudder }}\right) \frac{L_{\text {rudder }}}{I_{z}} \tilde{\delta}_{\text {rudder }}-\frac{C_{\text {rud }_{\mathrm{d}}} u_{\text {prop }} r}{I_{z}} \\
& \dot{\phi}=p+\left(q \sin \phi_{\text {ref }}+r \cos \phi_{r e f}\right) \theta_{\text {ref }} \\
& \dot{\theta}=q \cos \phi_{\text {ref }}-r \sin \phi_{\text {ref }}
\end{aligned}
$$

The equation for the heading or yaw $\psi$ is ignored, since the heading compensator is designed into the guidance logic.

Flight Control: The model of the airplane in flight can again be divided into four LTI systems. However, the systems are not fully decoupled. Depending on the roll angle, the rudder and elevator will influence pitch and lateral acceleration to different extents respectively. Forward velocity of the aircraft is influenced solely by the throttle. Similarly, roll is controlled by the ailerons. 
Full state feedback with an integrator is used to control the forward velocity of the airplane $v_{x_{B}}$ using throttle. The $z_{E}$-control loop uses full state feedback with an integrator term to produce a desired pitch angle $\theta_{\text {ref }}$. Depending on the roll angle of the airplane at a specific instant, a mixing of elevator and rudder is used to get the airplane to the desired pitch attitude. Lateral acceleration is commanded by the trajectory follower, which is detailed in Section VII. The lateral acceleration is produced by a mixing of elevator and rudder in the same manner as the pitch angle. The two contributions to elevator and rudder deflection are then aggregated to produce the true commands. The lateral acceleration reference $a_{c m d}$ is also used to produce a desired roll angle as

$$
\phi_{\text {ref }}=\arctan \left(\frac{a_{c m d}}{g}\right)
$$

With the assumption that the airplane is not ascending or descending rapidly, this reference will put the airplane in an attitude where only the elevator will be used to achieve the desired pitch angle and lateral acceleration, that is, in an attitude corresponding to what would be considered normal flight, where the acceleration along the $y_{B}$-axis is zero. All loops are controlled using a PID controller structure, with the exception of the lateral acceleration loop. This loop is controlled with a proportional gain only, since the trajectory follower itself emulates a PD controller.

\section{Transition Logic}

Transitioning the aircraft between flight regimes allows the vehicle to take advantage of its level flight capabilities and benefits (range, speed, etc.) while enabling the vehicle to operate and land in relatively small spaces using its hovering capabilities. However, enacting this transition can be difficult in a small space. For example, in order to transition from a hover to level flight, the controller must adjust the vehicle's throttle so as to allow the gravitational force acting on the airplane to pull the vehicle's nose down in a controlled descent, while trimming out its control surfaces to ensure that the vehicle has the heading, pitch, and roll angles to complete the transition successfully. Although executing transitions between flight modes is possible for a well-trained human pilot in large open spaces, transitions must be performed very efficiently in small spaces to enable the vehicle to recover both altitude and position control. This section describes how the transitions are performed automatically.

Transition to Flight: In order to transition to level flight in a confined space, the airplane needs to quickly gain speed while losing a minimal amount of altitude. This is achieved by using the hover controller. With an instantaneous movement of the reference waypoint to a distant location, in the direction of the desired transition, these goals can be met satisfactorily. As the airplane tries to move quickly to the new location, the pitch angle is reduced. By selecting the distant waypoint $15 \mathrm{~m}$ away from the hover location, the loss of altitude is small, while the airplane gains enough speed to meet the requirements of horizontal flight. The distant waypoint is used only to produce a rapid decrease in pitch angle; the controller switches into the level flight state after traveling only about $1 \mathrm{~m}$, where the reduction of pitch angle to less than $30^{\circ}$ required to switch is achieved. It was also found during flight tests that a $5 \%$ reduction in throttle from that required to maintain altitude during hover helped the airplane pitch forward slightly quicker. This improvement can be attributed to the propeller airflow lag that causes the airplane to resist the desired forward pitching moment. A larger reduction in throttle causes an even quicker drop in pitch. However, if the pitch of the airplane is brought down faster, the airplane loses more altitude. Hence, a balance between altitude loss and pitch rate has to be achieved.

Transition to Hover: The challenge of transitioning to hover is obviously the opposite of the challenges of transitioning to flight. As the airplane transitions to hover, altitude needs to be

12 of 18 
maintained, and the forward velocity needs to quickly go from level flight velocity to zero. In order to achieve the desired transition characteristics, the elevator and rudder are used in combination to minimize the time used to pitch the airplane to a nose up attitude. This rapid procedure puts the vehicle in a high angle of attack situation throughout the maneuver. Due to the dynamics of the airplane, a major portion of the forward velocity is lost to drag. To prevent the airplane from entering a complete stall, which would cause the aircraft's nose to pitch forward and compromise the desired transition, the throttle is set slightly lower than what is necessary to keep the airplane in a hover. This throttle setting ensures that full control authority is maintained for elevator and rudder by providing propeller airflow over the control surfaces. At the same time, most of the wing lacks coverage by the propeller airflow, which causes high drag from the stalled portions of the wing to quickly reduce the speed of the airplane. During transition to hover, the ailerons work in the same manner as during regular flight, that is, the ailerons are used to keep the airplane along the predefined trajectory leading up to the hover reference point.

The transition logic used to transition the airplane from level flight to hover is characterized by

$$
\begin{aligned}
\delta_{\text {elevator }} & =\cos (\phi) \delta_{\text {elevator max }_{\text {max }}} \frac{C_{L_{\delta_{\text {rud }}}} A_{\text {rudder }} \frac{L_{\text {rudder }}}{I_{z}}}{C_{L_{\delta_{\text {ele }}}} A_{\text {elevator }} \frac{L_{\text {elevator }}}{I_{y}}} \\
\delta_{\text {rudder }} & =\sin (\phi) \delta_{\text {rudder }_{\max }} \\
\delta_{\text {throttle }} & =k m g,
\end{aligned}
$$

where $k$ is a number slightly lower than unity. The aileron drag component is not included here, since the contribution to the total throttle is negligible in comparison with $m g$. Altitude hold is also not of significant interest during transition from level flight to hover.

As can be seen, transition to hover is done in an open-loop manner for all controls except for ailerons. The scaling on elevator is based on the assumption that the airplane will have a larger elevator control authority than rudder control authority. This is based on the fact that the elevator control surface is larger than the rudder control surface, while the maximum deflections of each is similar. This assumption was true for both airplane models used in our flight testing. The scaling factor ensures that the combination of elevator and rudder creates a pure pitching moment regardless of the roll angle of the airplane during transition to hover.

\section{Vehicle Guidance Logic}

The trajectory following logic is based on [10]. It works by selecting a reference point on the desired trajectory, at a predefined distance $L_{1}$ from the vehicle. Based on the angle $\eta$ between the vehicle velocity vector and a line from the vehicle to the reference point, a lateral acceleration command is calculated as illustrated in Figure 4. The lateral acceleration command $a_{c m d}$ is calculated as

$$
a_{c m d}=2 \frac{V^{2}}{L_{1}} \sin \eta
$$

Note that the trajectory follower will always try to move the vehicle in the direction of the $L_{1}$ line segment, hence pulling the vehicle

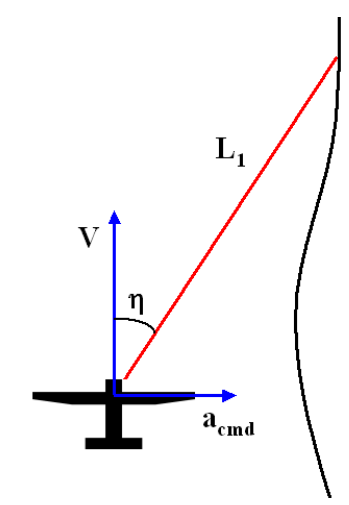

Figure 4. Trajectory following algorithm toward the desired trajectory. As the vehicle closes in on the trajectory, the vehicle is commanded to level out and approach the desired trajectory at a smaller angle. It is also readily shown that the trajectory follower produces a lateral acceleration command equal to the centripetal acceleration necessary to maintain a flight path on a circular segment by 

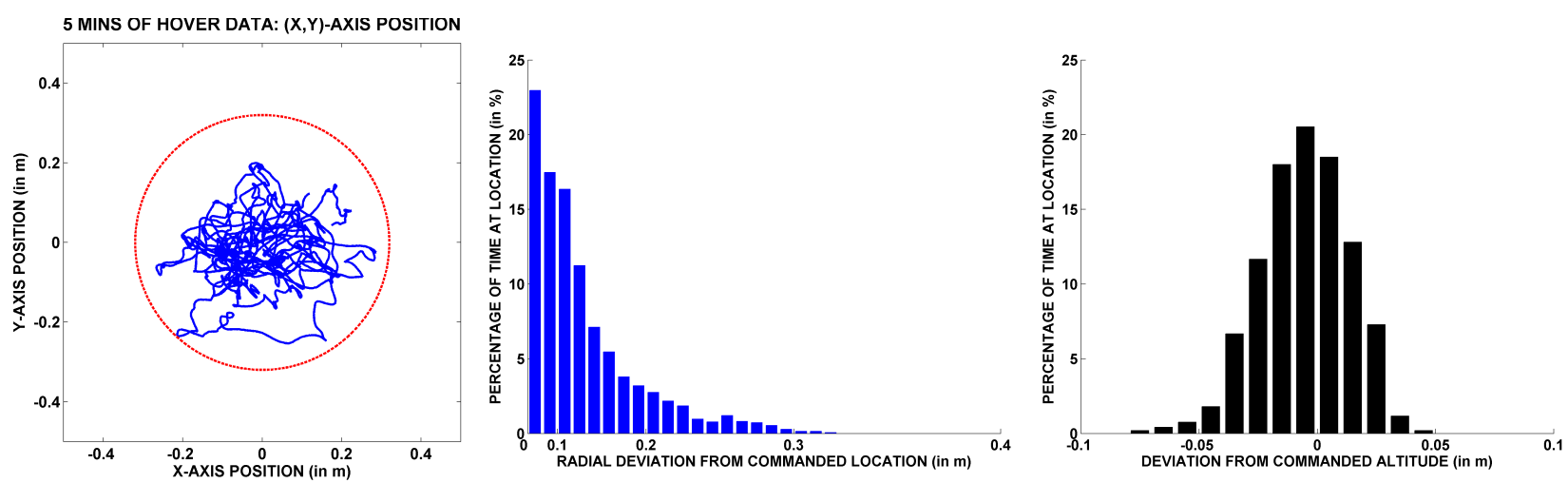

Figure 5. Airplane commanded to hover at $(x, y, z)=(0,0,1.5) \mathbf{m}$ for $\mathbf{5}$ min: $x-y$ plot of vehicle position (left), histograms showing the radial distance error for the vehicle from $(x, y)=(0,0) \mathrm{m}$ (middle) and altitude error from $z=1.5 \mathrm{~m}$ (right)

noting that $L_{1}=2 R \sin \eta$. This relationship shows an inherent limit in the choices of $L_{1}$, since the minimum radius of a circle that can be followed by the trajectory follower is $R_{\min }=L_{1} / 2$. The radius limitation is also made apparent by realizing that with the assumption that the vehicle is on the desired trajectory, no reference point on a circle can be selected if the reference distance $L_{1}$ is larger than the diameter of the circle that the vehicle is trying to follow.

Assuming that the above limitation on $L_{1}$ is not exceeded, the choice of $L_{1}$ is arbitrary. However, due to the manner in which the trajectory follower works, a small $L_{1}$ effectively makes the controller more aggressive, whereas a larger $L_{1}$ gives a smoother vehicle response. Empirical testing has shown that the controller performance is heavily influenced by the choice of $L_{1}$. An $L_{1}$ value of $2.4 \mathrm{~m}$ was utilized for the trajectory following flight demonstrations presented in this paper. As with any implementation of this tracking algorithm, it is important to have an efficient way of computing the desired trajectory and the intersection point of the $L_{1}$ line and the desired trajectory. Various heuristics can be used to pick a target point in the case that an intersection point does not exist.

\section{Results}

Numerous hover and level flight tests were performed using the Blade 3D and Ikarus Yak 54 Shock Flyer foam aircrafts in the RAVEN at MIT. ${ }^{7}$ First, typical results are shown in Figure 5 in which the vehicle was commanded to hold its position at $\left(x_{E}, y_{E}, z_{E}\right)=(0,0,1.5) \mathrm{m}$ for five minutes. Figure 5 shows three plots, including a plot of the vehicle $x-y$ location while it maintained its position and attitude. The dashed red circle in the picture has a radius of $0.32 \mathrm{~m}$. This circle would correspond to the allowable movement in a $1.50 \mathrm{~m}$ wide corridor or equivalent, based on the vehicle's wingspan. As shown in Figure 5, the vehicle maintained its position inside this circle during the five-minute test period. The remaining plots give histograms of the vehicle's $x-y$ and $z$ positions. The first histogram shows the radial distance error for the vehicle from $(x, y)=(0,0) \mathrm{m}$, while the second histogram shows the vehicle's altitude error from $z=1.5 \mathrm{~m}$. These plots confirm that the vehicle was within a $20 \mathrm{~cm}$ circle for over $87 \%$ of the 5 minute flight. These plots also show that the vehicle precisely maintained its altitude (staying between 1.4 to $1.6 \mathrm{~m}$ ) during the entire hover test.

Next, since the airplanes are autonomous from take-off to landing, the aircraft takes off and lands in a perching configuration. Figure 6 shows a photographed sequence of the airplane perching on a fixed cement pole located in the middle of the RAVEN flight space in hover mode. Test flight results for five consecutive take-off and landing sequences are shown in Figures 7 and 8. In this test, the landing pad on the cement pole is located at $(\mathrm{x}, \mathrm{y}, \mathrm{z})=(0,2.67,1.03) \mathrm{m}$ in the room and 

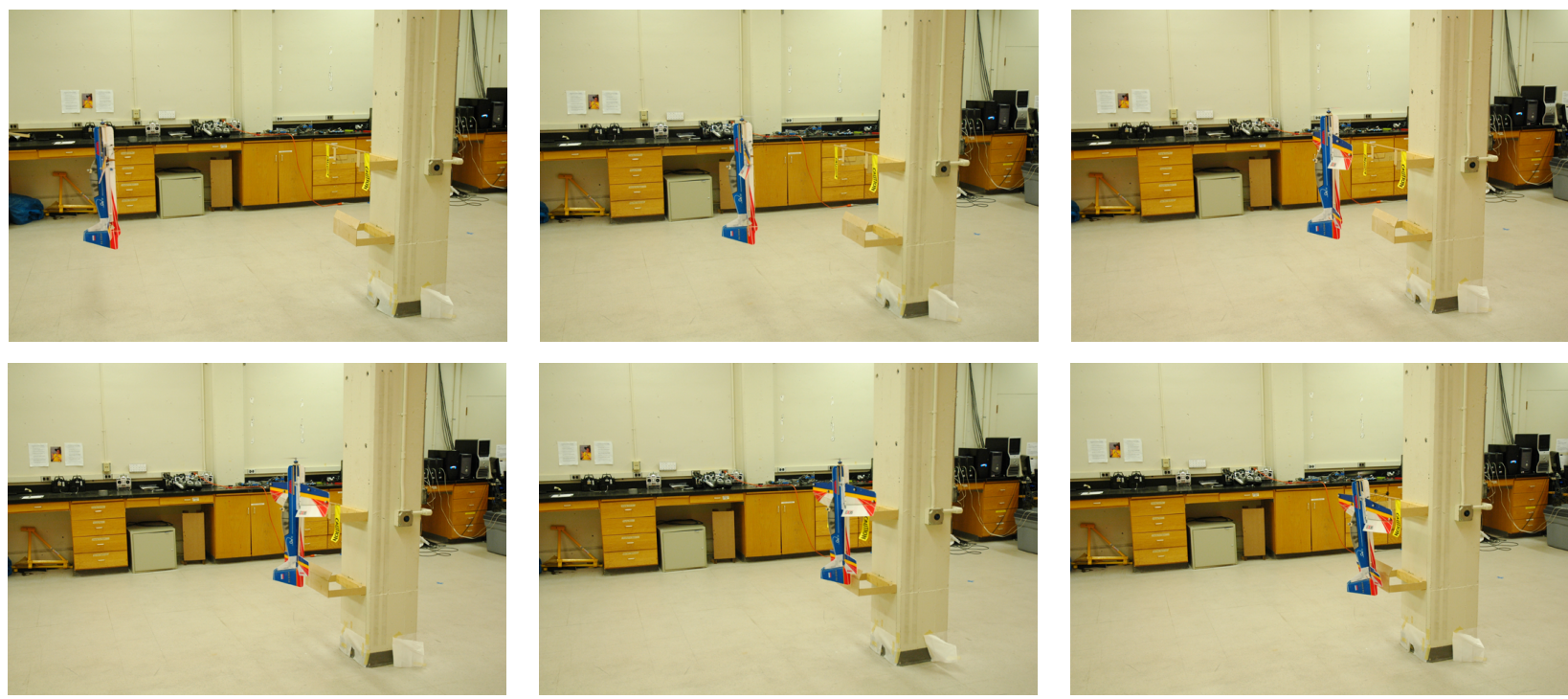

Figure 6. Autonomous landing in hover mode - perching on the fixed landing platform on a pole
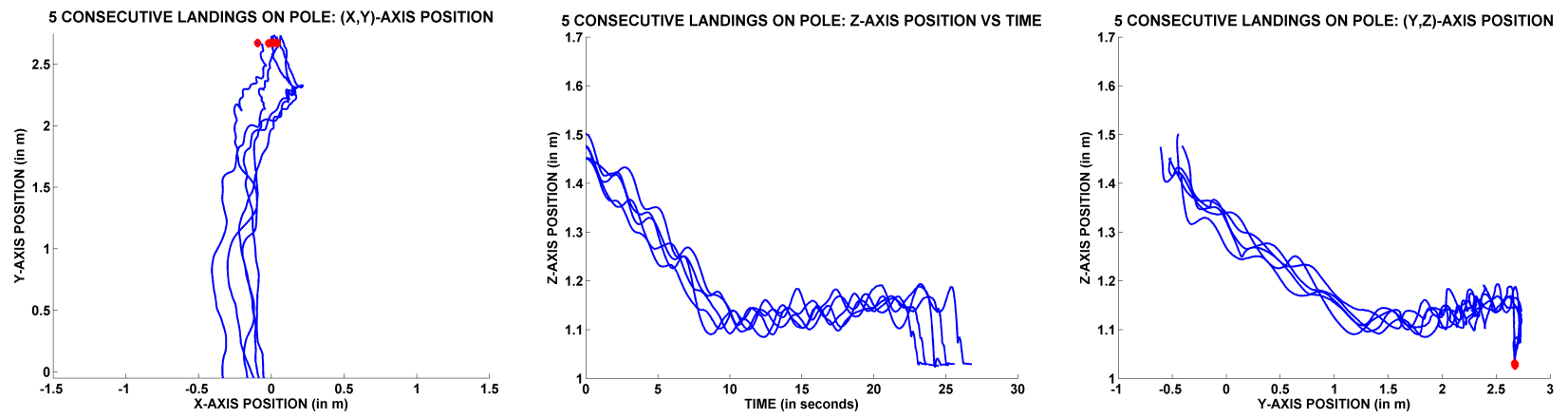

Figure 7. Five consecutive landings - Airplane commanded to perch on the cement pole landing apparatus at $(x, y, z)=(0,2.67,1.03) \mathbf{m}: x-y$ plot of vehicle position (left), $z$-axis position (middle), and $y-z$ plot of vehicle position (right)
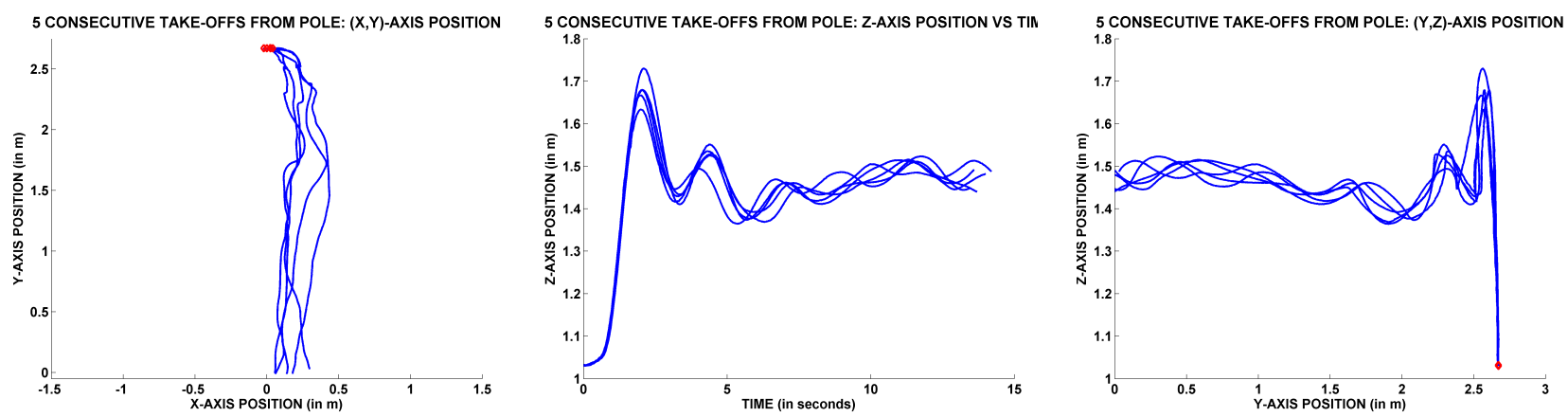

Figure 8. Five consecutive take-offs - Airplane commanded to take-off from previous landing position on cement pole at $(x, y, z)=(0,2.67,1.03) \mathbf{m}: x-y$ plot of vehicle position (left), $z$-axis position (middle), and $y-z$ plot of vehicle position (right) 

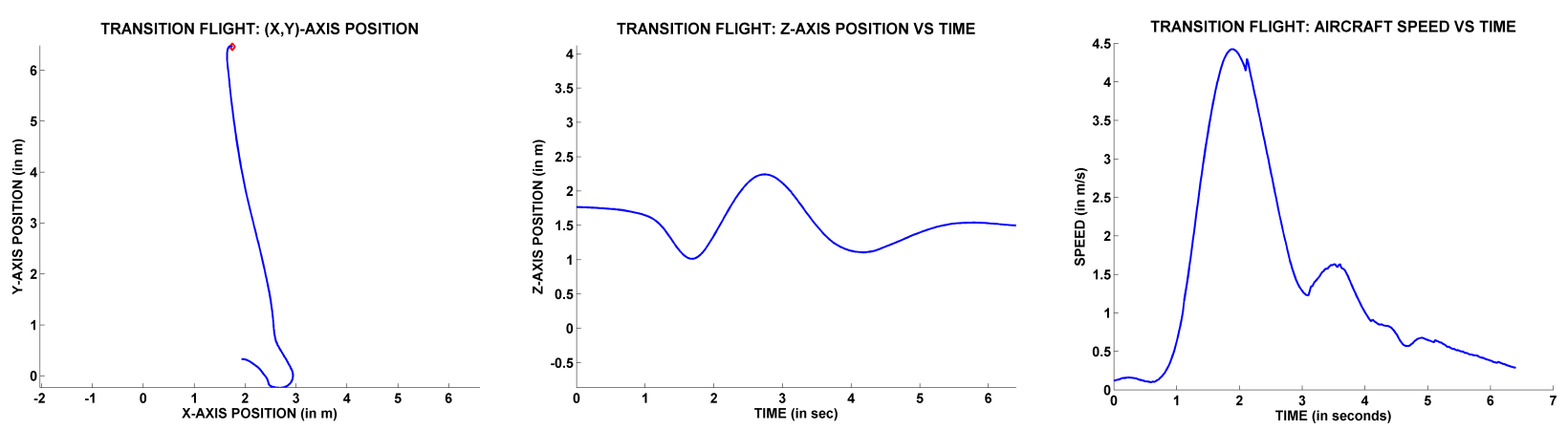

Figure 9. Transition test - Starting from a hover, transition to and from level flight mode: $x-y$ plot of vehicle position (left), $z$-axis position (middle), and vehicle speed (right)

the vehicle was commanded to take off, hover for about $15 \mathrm{~s}$ and then attempt another landing. In these plots, the vehicle begins its approach near $(\mathrm{x}, \mathrm{y})=(0,0,1.5) \mathrm{m}$ and moves toward the landing platform in the hover mode. As it approaches the landing location, the vehicle reduces the altitude to be just above the landing site. Then, once the vehicle detects that it is in the proper location with a correct heading, the vehicle descends into the landing location to await the next take-off command. After the take-off command is given, the throttle is accelerated to pull the vehicle directly above the landing platform before moving the vehicle away from the pole.

In Figures 7 and 8 the red diamonds at the end of each trajectory show the landing/takeoff location of the vehicle. Throughout the entire sequence, the fully-autonomous vehicle was commanded to take off from the location where it had landed. Note that all of these take-off locations are clustered within $0.07 \mathrm{~m}$ of each other. Likewise all of the landing locations are within $0.15 \mathrm{~m}$ of each other (in fact the first four landings are clustered within $0.07 \mathrm{~m}$ of each other, but the fifth landing was on the left-hand part of the landing apparatus). Also note that the vehicle's paths into and off the platform are very consistent during each landing and take-off. During take-off, there is an overshoot as the vehicle pulls itself off the platform which is a result of a safety feature designed to ensure that the vehicle stays away from the pole.

Before attempting level flight tests with the aircraft, the vehicle's transition logic was also demonstrated. Figure 9 shows data from one of these transition tests in which the vehicle autonomously moved from the hover take-off location near the pole to the Northeastern area of the flight space. The test began from the red diamond and flew south. First, note that this test occurred in under $6 \mathrm{~s}$. In fact, each vehicle transition takes approximately $1.5 \mathrm{~s}$ to complete. As the vehicle transitions to level flight mode the vehicle loses altitude while picking up speed. In this test after the vehicle achieves the level flight mode (i.e., the vehicle's calculated pitch angle is less than 30 degrees), the vehicle is immediately commanded back to hover mode. Notice that as the vehicle begins to pull out of the level flight state, the vehicle gains speed until the system reduces the throttle control as the vehicle gains altitude. In addition, after the vehicle exceeds the desired hover altitude of $1.5 \mathrm{~m}$, the vehicle's velocity reduces quickly and the vehicle's calculated pitch increases dramatically. In fact, the pitch angle passes 90 degrees as the vehicle tries to reduce speed and altitude quickly to regain the hover control.

Finally, a flight demonstrating the full capabilities (hover to level flight, fly 3 laps, transition to hover) was demonstrated. Figure 10 shows data from two of these three-lap tests. Figure 11 shows images a various stages of the flight. Once again, the vehicles autonomously moved from the hover take-off location near the pole to the northeastern area of the flight space shown in Figure 10. From this location, the vehicle transitioned to level flight mode, flew three laps around the pole (as shown in the $x-y$ location plot in Figure 10) and then transitioned back to the hover state before attempting a landing. During the level flight in the RAVEN, the vehicle maintains a speed 

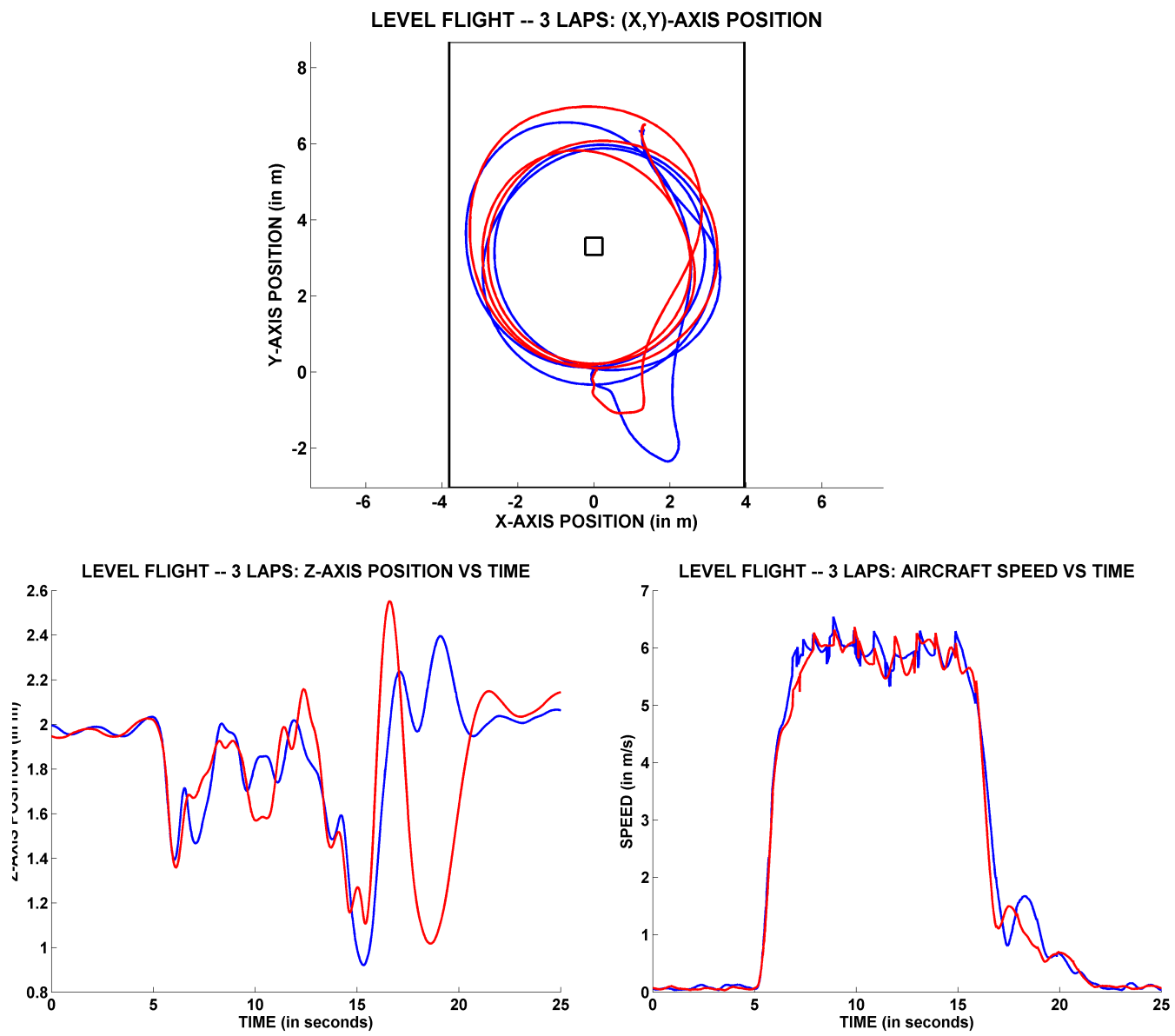

Figure 10. Level flight test in constrained environment - Starting from a hover, the vehicle transitions to level flight mode, flies three laps around the room and then transitions back to hover mode: $x-y$ plot of vehicle position (top), $z$-axis position (bottom left), and vehicle speed (bottom right). The red and blue plots represent two separate flight tests.
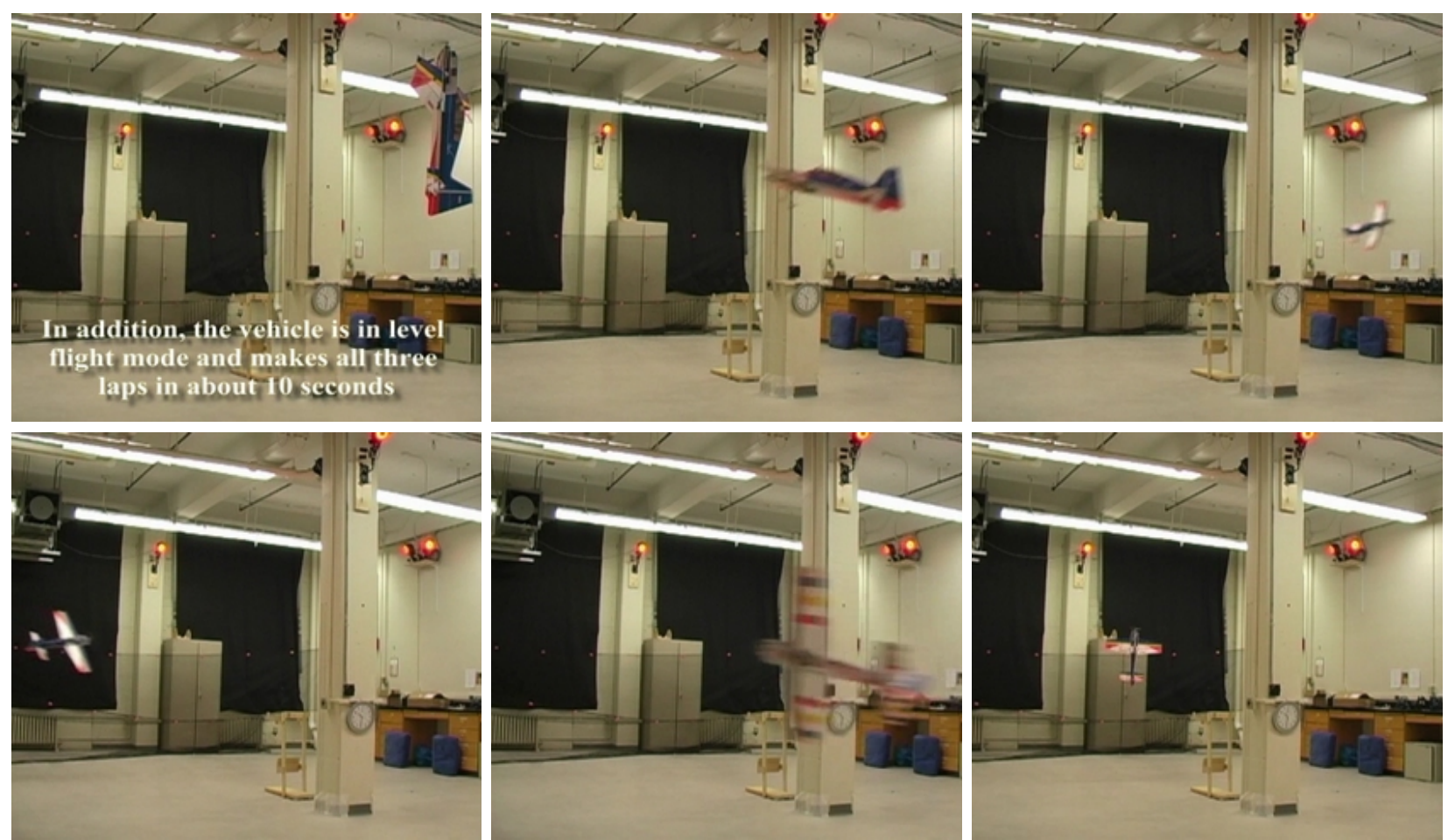

Figure 11. Autonomous aircraft hover, transition to level flight and back to hover. 
of approximately $6 \mathrm{~m} / \mathrm{s}$ during each of the three laps. In addition, the vehicle is flying a circular route with a radius of about $2 \mathrm{~m}$. Therefore, the three laps take about $10 \mathrm{~s}$ from the time the vehicle enters level flight mode to the time the vehicle transitions back to the hover state. A flight from one of these tests can be seen at http://aerobatics.mit.edu.

\section{Conclusion}

This paper presents both vehicle models and test flight results for an autonomous fixed-wing airplane that is designed to take-off to a hover, transition to and from traditional fixed-wing level flight modes, and perch on a vertical landing platform in a highly space constrained environment. The flight control for this commercially available fixed-wing model airplane are achieved using the motion capture system available in the MIT Real-time indoor Autonomous Vehicle test ENvironment (RAVEN). These experiments represent the first known flight results in the literature of autonomous aggressive indoor flight using a fixed-wing R/C aircraft. Future work will expand on these test to investigate landing on moving objects in a hover and performing other aerobatic maneuvers in the indoor test environment.

\section{Acknowledgments}

The authors would like to thank Spencer Ahrens, Brett Bethke, Luc Brunet, Eli Cohen, Daniel Dale, Brandon Luders, and Adam Woodworth for their invaluable assistance. Research supported by the Boeing Company, Phantom Works, Seattle and by AFOSR grant FA9550-04-1-0458.

\section{References}

${ }^{1}$ B. L. Stevens and F. L. Lewis. Aircraft Control and Simulation, Second Edition. J. W. Wiley and Sons, 2003.

${ }^{2}$ D. Biermann and R. N. Conway. Propeller Charts for the Determination of the Rotational Speed for the Maximum Ratio of the Propeller Efficiency to the Specific Fuel Consumption. Technical report, National Advisory Committee on Aeronautics, 1942.

${ }^{3}$ E. N. Johnson, M. A. Turbe, A. D. Wu, S. K. Kannan, and J. C. Neidhoefer. Flight Test Results of Autonomous Fixed-Wing UAV Transitions to and from Stationary Hover. In Proceedings of the AIAA Guidance, Navigation, and Control Conference Exhibit, Monterey, CO, August 2006.

${ }^{4}$ E. Frazzoli. Robust Hybrid Control for Autonomous Vehicle Motion Planning. Department of aeronautics and astronautics, Massachusetts Institute of Technology, Cambridge, MA, June 2001.

${ }^{5}$ A. J. Hanson. Visualizing Quaternions. Morgan Kaufman Publishers, San Francisco, CA, 2006.

${ }^{6}$ J. B. Kuipers. Quaternions and Rotation Sequences. Princeton University Press, Princeton, NJ, 1999.

${ }^{7}$ M. Valenti, B. Bethke, G. Fiore, J. How, and E. Feron. Indoor multi-vehicle flight testbed for fault detection, isolation, and recovery. In Proceedings of the AIAA Guidance, Navigation, and Control Conference and Exhibit, Keystone, CO, August 2006.

${ }^{8}$ N. Knoebel, S. Osborne, D. Snyder, T. Mclain, R. Beard, and A. Eldredge. Preliminary modeling, control, and trajectory design for miniature autonomous tailsitters. In Proceedings of the AIAA Guidance, Navigation, and Control Conference and Exhibit, Keystone, CO, August 2006.

${ }^{9}$ R. H. Stone. Control Architecture for a Tail-Sitter Unmanned Air Vehicle. In Proceedings of the 5th Asian Control Conference, Melbourne, Australia, July 2004.

${ }^{10} \mathrm{~S}$. Park, J. Deyst, and J. P. How. A New Nonlinear Guidance Logic for Trajectory Tracking. In Proceedings of the AIAA Guidance, Navigation, and Control Conference, Providence, RI, August 2004.

${ }^{11}$ University of Sydney. T-Wing Aircraft Homepage. Avaiable at http://www.aeromech.usyd.edu.au/uav/twing/, October 2006.

${ }^{12}$ M. Valenti, B. Bethke, D. Dale, A. Frank, J. McGrew, S. Ahrens, J. P. How, and J. Vian. The MIT Indoor Multi-Vehicle Flight Testbed. In Proceedings of the 2007 IEEE International Conference on Robotics and Automation (ICRA '07), Rome, Italy, April 2007. Video Submission.

${ }^{13}$ W. E. Green and P. Y. Oh. A MAV That Flies Like an Airplane and Hovers Like a Helicopter. In Proceedings of the 2005 IEEE/ASME International Conference on Advanced Intelligent Mechatronics, Monterey, CA, July 2005.

${ }^{14}$ W. F. Chana and J. F. Coleman. World's First VTOL Airplane Convair/Navy XFY-1 Pogo. In Proceedings of the International Powered Lift Conference, pages 99-104, Jupiter, FL, November 1996.

18 of 18 